Background In 2012 changes in the home oxygen service (HOS) contract offered patients the potential to benefit from new technology designed to assist ambulatory oxygen (AO) use, such as liquid oxygen (LOX) and refillable cylinders (Homefill). Prior to the change in contract only three services were thought to be commissioned in London (serving approximately 10,000 HOS users, costing $£ 10.5 \mathrm{~m}$ ), with many areas attempting to meet increasing demand with no increase in resources.

Aim

- To determine the service provision, commissioning arrangement and assessment protocols for AO across London

- Establish an AO network across London

Methods A telephone audit was carried out in January-March 2013 with all known oxygen assessment centres in London. Two clinicians used an agreed proforma, with email follow-up. The interview included questions regarding; commissioning/funding; location; access to service, referrals and pathway; assessment protocol; disciplines/grades; and integration with respiratory services.

Results 34 interviews were performed across the 32 London boroughs. Key findings are:

Access: Two boroughs had no service, some had multiple.

Who: In 20 teams nursing staff assessed; 15 teams, physiotherapists; and 7 teams, respiratory physiologists.

Where: 16 assessed in the hospital, 9 in the community and 7 in both.

How: The majority (94\%) performed the 6MWT, however teams that assessed in the home did not use validated reproducible exercise tests.

Equipment for assessment: The majority had standard cylinders (88\%); 53\% had lightweight and conservers; and other devices were rarely (3-13\%) available.

Size: 16 services (47\%) carried out less than 5 assessments per month.

Funding: 47\% have some arrangements in place, $29 \%$ had no funding or no service provided and $24 \%$ unclear.

Integration: $67 \%$ were part of an integrated service, $18 \%$ stand alone, $12 \%$ unclear and $6 \%$ had no service.

Conclusions Service provision for AO across London is varied, with no standardised referral pathway, assessment protocol and often limited range of equipment available for assessment. This raises concerns over access to services, clinical assessment skills/ competencies and unsuitable prescriptions. Approximately half of the services have no or unclear funding arrangements and although the majority of services (67\%) are integrated within a wider COPD/IRS there was no established network and many clinicians felt isolated.

\section{M26 TEACHING STUDENTS TO PRESCRIBE OXYGEN SAFELY: THE IMPACT OF AN E-LEARNING MODULE AND BTS OXYGEN PRESCRIPTION SECTION ON OXYGEN PRESCRIBING BY MEDICAL STUDENTS TAKING FINAL MBBS EXAMINATIONS}

${ }^{1} \mathrm{D}$ Hammersley, ${ }^{2} \mathrm{LE}$ Smith, ${ }^{2} \mathrm{D}$ Furmedge, ${ }^{1} \mathrm{C}$ Ward, ${ }^{1} S$ Kennedy, ${ }^{1} \mathrm{U}$ Restrick, ${ }^{2} \mathrm{~A}$ Sturrock; ${ }^{1}$ Department of Respiratory Medicine, Whittington Health, London, England; ' 2 University College London Medical School, London, England

\subsection{6/thoraxjnl-2013-204457.436}

Introduction and Objectives National audits show oxygen prescribing is not consistently safe, despite BTS Emergency Oxygen
Guidelines. We previouslyidentified that medical students were unable to safely prescribe oxygen at final MBBS examinations. ${ }^{1}$ A compulsory e-Learning module was introduced to address this unmet educational need. We assessed the impact of this intervention in 2013.

Methods An Oxygen Prescribing Final MBBS OSCE station was used in 2012 and 2013. In 2013 candidates (350) completed a new compulsory oxygen e-Learning module. Candidates in 2012 (227) had not. In 2013 the exam prescription chart also included an oxygen prescription section. Each year, candidates were presented with one of two clinical scenarios. Scenario 1: 72-yearold patient with COPD, and Scenario 2: 72-year-old hypoxic patient without respiratory disease. Oxygen prescriptions were assessed against BTS standards across a number of domains. They were classified as 'safe/unsafe' and 'perfect/imperfect' by a respiratory nurse specialist.

Results Some improvements were seen in both scenarios (See Table 1), particularly prescription of the correct target saturation range. In $201240 \%$ (42/105) prescribed correct range for the non-COPD scenario; in 2013 this was $98 \%$ (154/156).

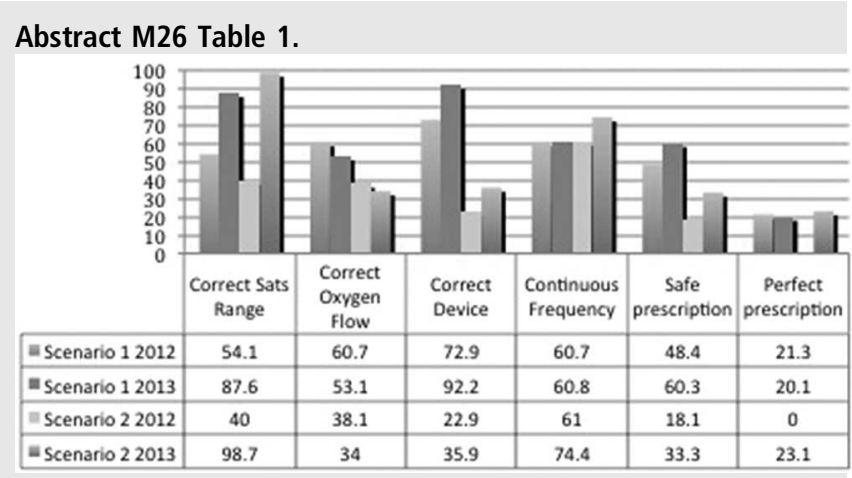

Conclusions Introducing an oxygen e-Learning module and BTS-recommended oxygen prescription section resulted in improved competence and safety of oxygen prescribing with significant improvement in correct target saturation ranges. However, students still have gaps in equipment knowledge and a high proportion did not prescribe oxygen safely for a patient without respiratory disease. The e-Learning module was undertaken by students at a point close to examinations; moving this earlier in the year may lead to better engagement and improve the understanding of oxygen prescribing in non-COPD patients, emphasised in the module. Safer prescribing is enabled by oxygen prescription sections with target range saturation choices but equipment education is also needed. Adverse consequences of incorrect oxygen use continue to cause patients harm. Ensuring undergraduates have the practical knowledge and skills to prescribe oxygen safely is essential.

\section{REFERENCE}

1. D Hammersley, A Connor, C Ward, et al. Competence in, and safety of, oxygen prescribing by medical students taking Final MBBS as assessed by Objective Structured Clinical Examination. Thorax 2012; 67(Suppl 2):A168

\section{M27 SHOULD THERE BE A RESPIRATORY-SPECIFIC MODIFIED EARLY WARNING SCORE?}

H Finnamore, M Pritchard, O Abdul Kadhir, J Mayer, J Bannon, H Burhan; Royal Liverpool and Broadgreen University Hospitals NHS Trust, Liverpool, UK

10.1136/thoraxjnl-2013-204457.437 
Introduction The Modified Early Warning Score (MEWS) was developed and validated as an objective scoring system to aid healthcare staff in identifying patients at risk of "catastrophic deterioration" in the acute hospital setting ${ }^{(1)}$. At the Royal Liverpool and Broadgreen University Hospitals NHS Trust (RLBUHT), the Acute Response Team (ART) is led by advanced nurse practitioners, who respond to calls when patients have a MEWS of 4 or more. It was noted that a large proportion of calls were to respiratory patients, many requiring no intervention.

Methods Details of every ART call to medical patients throughout $2012(\mathrm{n}=883)$ were recorded on clinical proforma and collated on an Excel database. Data were analysed using STATA 12, as part of a service evaluation. Outcomes measured were: numbers of ART calls made to respiratory and remaining medical wards, numbers of Do Not Attempt Resuscitation (DNAR) orders in place, MEWS, investigations performed by the ART, critical care transfer and the 7 and 30 day mortality.

Results The 53 respiratory beds account for only 14\% of the medical bed-base but generated 25\% of ART calls. Respiratory patients scored more highly on respiratory rate (RR) and oxygen saturations $\left(\mathrm{SpO}_{2}\right)$ MEWS parameters than other medical patients. ART investigation rates were similar in all patients but only $1 \%$ were transferred from respiratory to critical care. There were more DNAR orders and both 7 and 30 day mortality were higher on the respiratory wards (see Table 1 ).

Abstract M27 Table 1. Summary of ART calls for all of medicine, medicine (not respiratory) and respiratory only.

\begin{tabular}{llll} 
Variable & All medicine & Medicine (not resp) & Respiratory \\
\hline ART call total n, (\%) & $883(100)$ & $663(75.1)$ & $220(24.9)$ \\
$\begin{array}{l}\text { DNAR in place } \\
\text { MEWS }\end{array}$ & $206(23.3)$ & $135(20.4)$ & $71(32.3)$ \\
MEWS (Respiratory Rate & & & \\
$\mathbf{0}$ & $194(22.3)$ & $158(24.2)$ & $36(16.7)$ \\
$\mathbf{1}$ & $286(32.9)$ & $213(32.6)$ & $73(33.8)$ \\
$\mathbf{2}$ & $177(20.4)$ & $130(19.9)$ & $47(21.7)$ \\
$\mathbf{3}$ & $206(23.7)$ & $147(22.5)$ & $59(27.3)$ \\
MEWS (Sp02) & & & $13(6.0)$ \\
$\mathbf{0}$ & $112(13.0)$ & $99(15.3)$ & $38(17.5)$ \\
$\mathbf{1}$ & $252(29.2)$ & $214(33.1)$ & $58(26.7)$ \\
$\mathbf{2}$ & $203(23.5)$ & $145(22.4)$ & $107(49.3)$ \\
$\mathbf{3}$ & $296(34.6)$ & $189(29.2)$ & $1(1.0)$ \\
ITU/HDU referral & $18(2.0)$ & $16(2.4)$ & $8(3.6)$ \\
BiPaP commenced & $10(1.1)$ & $2(0.3)$ & $73(33.2)$ \\
$\mathbf{7}$ day mortality & $237(26.8)$ & $164(24.7)$ & $76(36.0)$ \\
30 day mortality & $239(27.1)$ & $183(28.0)$ & \\
(cumulative) & & & \\
\hline & & &
\end{tabular}

Discussion Many respiratory patients score highly on RR and $\mathrm{SpO}_{2}$ MEWS parameters due to their chronic disease. The increased use of DNAR orders in respiratory patients reflects a greater burden of chronic disease and therefore a poorer prognosis. This may explain the low rates of transfer to critical care and high mortality rates. We suggest a respiratory-specific MEWS may reduce ART calls to stable respiratory patients and, for respiratory patients with DNAR orders, automatic exemption from ART calls should be considered.

\section{REFERENCES}

1. Subbe, Kruger and Rutherford. QJM (2001) 94 (10): 521-526.

\section{M28 FROM NEWS TO CREWS: THE CHRONIC RESPIRATORY EARLY WARNING SCORE FOR PATIENTS WITH CHRONIC HYPOXAEMIA}

${ }^{1}$ SR Eccles, ${ }^{2} \mathrm{C}$ Subbe, ${ }^{3} \mathrm{D}$ Hancock, ${ }^{3} \mathrm{~N}$ Thompson; ${ }^{1}$ Wrexham Maelor Hospital, Wrexham, Wales; ${ }^{2} Y$ sbyty Gwynedd, Bangor, Wales; ${ }^{3}$ Cardiff University School of Medicine, Cardiff, Wales $^{3}$

\subsection{6/thoraxjnl-2013-204457.438}

Introduction In patients with respiratory conditions associated with chronic hypoxaemia such as COPD, use of the National Early Warning Score (NEWS)[1] leads to a high number of triggers. We designed a simple variant of NEWS for patients with chronic hypoxaemia.

Methods Data was collected from respiratory wards at two hospitals in North Wales over a three-month period. Patients were categorised into those with chronic hypoxaemia $(\mathrm{CH}$, target oxygen saturations $88-92 \%)$ and others $(\mathrm{O}$, target saturations $94-$ 98\%). Vital signs were recorded on admission to hospital, at the peak NEWS score during admission, and during a period of stability/at discharge.

Results 196 admissions were included in the analysis. 98 (50\%) were male, 91 had COPD, 26 received long-term oxygen therapy. Mean age was 70 (range 19-102). 78 patients had target saturations of $88-92 \%$ (CH patients). The mean NEWS for all patients on admission was 5 (SD 3), mean peak NEWS was 7 (SD 3), and median hospital length of stay was 10 days (IQR 6:17).

23 patients died within 30 days; 12 with $\mathrm{CH}$. Of these, all patients with $\mathrm{CH}$ scored $6+$ using NEWS compared to $9 / 11 \mathrm{O}$ patients. When stable/at discharge, $32 \%$ of $\mathrm{CH}$ patients scored 6 + using NEWS (Welsh trigger) and 50\% scored $5+$ (RCP trigger). By using a recalibrated scoring system (CREWS, table 1), patients triggering during stability/at discharge were reduced to $14 \%(5+$ trigger $)$ and $8 \%(6+$ trigger $)$. All patients who subsequently died still triggered at peak CREWS scores.

Conclusion Patients with chronic respiratory conditions have altered baseline parameters that lead to a high number of NEWS triggers. CREWS reduces triggers, which may reduce unnecessary reviews and alarm fatigue, without compromising safety.

\section{REFERENCE}

1. Royal College of Physicians. National Early Warning Score (NEWS): Standardising the assessment of acute illness severity in the NHS. Report of a working party. London: RCP, 2012

Abstract M28 Table 1. Scores allocated for oxygen saturations (\%) for NEWS, and for CREWS for CH patients.

\begin{tabular}{lllll}
\hline & \multicolumn{5}{c}{ Score for oxygen saturations } \\
\cline { 2 - 5 } Scoring system & $\mathbf{3}$ & $\mathbf{2}$ & $\mathbf{1}$ & $\mathbf{0}$ \\
\hline NEWS & $\leq 91$ & $92-93$ & $94-95$ & $\geq 96$ \\
CREWS & $\leq 85$ & $86-87$ & $88-89$ & $\geq 90$ \\
\hline
\end{tabular}

\section{M29 NATIONAL EARLY WARNING SCORE (NEWS): IS IT BAD NEWS FOR OUR PATIENTS?}

E Cheetham, R Pais, M Wijesinghe; Royal Cornwall Hospital NHS Trust, Truro, United Kingdom

\subsection{6/thoraxjnl-2013-204457.439}

Background Titrated oxygen has been shown to significantly reduce mortality in patients presenting with acute exacerbations 